\title{
Sliding disks in the plane
}

\author{
Sergey Bereg ${ }^{1}$, Adrian Dumitrescu ${ }^{2}$, and János Pach ${ }^{3 \star}$ \\ 1 Computer Science, University of Texas at Dallas \\ P.O. Box 830688, Richardson, TX 75083, USA \\ besp@utdallas.edu \\ 2 Computer Science, University of Wisconsin-Milwaukee \\ 3200 N. Cramer Street, Milwaukee, WI 53211, USA \\ ad@cs. uwm. edu \\ 3 Courant Institute of Mathematical Sciences \\ 251 Mercer Street, New York, NY 10012-1185, USA \\ pach@cims.nyu.edu
}

\begin{abstract}
Given a pair of start and target configurations, each consisting of $n$ pairwise disjoint disks in the plane, what is the minimum number of moves that suffice for transforming the start configuration into the target configuration? In one move a disk slides in the plane without intersecting any other disk, so that its center moves along an arbitrary (open) continuous curve. We discuss efficient algorithms for this task and estimate their number of moves under different assumptions on disk radii and disk placements. For example, with $n$ congruent disks, $\frac{3 n}{2}+O(\sqrt{n \log n})$ moves always suffice for transforming the start configuration into the target configuration; on the other hand, $\left(1+\frac{1}{15}\right) n-O(\sqrt{n})$ moves are sometimes necessary.
\end{abstract}

\section{Introduction}

Consider a set (system) of $n$ pairwise disjoint objects in the plane that need to be brought from a given start (initial) configuration $S$ into a desired goal (target) configuration $T$. The motion planning problem for such a system is that of computing a sequence of object motions (schedule) that achieves this task. Depending on the existence of such a sequence of motions, we say that the problem is feasible or respectively, infeasible. Here we restrict ourselves to systems of disks with pairwise disjoint interiors, as objects, and moves that slide a disk without intersecting any other disk throughout the motion. The disks are not labeled, therefore if there exist congruent disks in the system, occupying any of the target positions with a congruent disk is allowed.

It is easy to see that, for the class of disks, the problem is always feasible. More generally, it is also feasible for the class of all convex objects, using sliding moves (Theorem 1 below). This old result appears in the work of Fejes Tóth and Heppes [8], but it can be traced back to de Bruijn [5]; the algorithmic aspects

\footnotetext{
* Supported by NSF grant CCR-00-98246, by an NSA grant, a PSC-CUNY Research
} Award, and grant OTKA-T-032-452 from the Hungarian Science Foundation. 
of the problem have been studied by Guibas and Yao [9]. We refer to this set of motion rules (moves) as the sliding model. Other reconfiguration rules (models) for systems of disks have been examined recently, for example: in [6] moves are restricted so that a disk can only be placed in a position where it is adjacent to at least two other disks; in [1] moves are translations along a fixed direction at each step. Reconfiguration for modular systems acting in a grid-like environment, and where moves must maintain connectivity of the whole system has been recently addressed in [7].

Theorem 1. Any set of $n$ convex objects in the plane can be separated via translations all parallel to any given fixed direction, with each object moving once only. If the topmost and bottommost points of each object are given (or can be computed in $O(n \log n)$ time), an ordering of the moves can be computed in $O(n \log n)$ time.

The following simple universal algorithm that can be adapted to any set of $n$ convex objects performs $2 n$ moves for reconfiguration of $n$ disks. In the first step ( $n$ moves), in decreasing order of the $x$-coordinates of their centers, slide the disks initially along a horizontal direction, one by one to the far right. Note that no collisions can occur. In the second step ( $n$ moves), bring the disks "back" to target positions in increasing order of the $x$-coordinates of their centers. (General convex objects need rotations and translations in the second step). Already for the class of disks, one cannot do much better in terms of the number of moves (see Theorem 3). For the class of segments (as objects), it is easy to construct examples that require $2 n-1$ moves for reconfiguration, even for congruent segments.

A move is a target move if it slides a disk to a final target position. Otherwise, it is a non-target move. Our lower bounds use the the following argument: if no target disk coincides with a start disk (so each disk must move), a schedule with $x$ non-target moves consists of at least $n+x$ moves.

Our paper is organized as follows. In Section 2 (Theorem 2), we estimate the number of necessary moves for the reconfiguration of systems of congruent disks. In Section 3 (Theorem 3), we estimate the number of necessary moves for the reconfiguration of systems of disks of arbitrary radii.

\section{Congruent disks}

We now consider reconfiguring sets of congruent disks in the plane. First, we prove the existence of a line bisecting the set of centers of the start disks such that the strip of width 6 around this line contains a small number of disks. A slightly weaker statement guaranteeing the existence of a bisecting line that cuts through few disks was given by by Alon et. al [2]. We have included our almost identical proof for completeness.

Lemma 1. Let $S$ be a set of $n$ pairwise disjoint unit (radius) disks in the plane. Then there exists a line $\ell$ that bisects the centers of the disks such that the parallel 
strip of width 6 around $\ell$ (that is, $\ell$ runs in the middle of this strip) contains entirely at most $O(\sqrt{n \log n})$ disks.

Proof. Set $m=c_{2} \sqrt{n \log n}$ where $c_{2}>0$ is a suitable large constant to be chosen later. Assume for contradiction that the strip of width $w=6$ around each line bisecting the set of centers of $S$ contains at least $m$ disks. Set $k=\lceil\sqrt{n / \log n}\rceil$ and consider the $k$ bisecting lines that form angles $i \theta$ with the positive direction of the $x$-axis (in counterclockwise order), where $i=0, \ldots, k-1$, and $\theta=\pi / k$.

Let $A_{i}$ be the set of disks contained (entirely) in the $i$-strip of width $w=6$ around the $i$ th bisecting line, $i=0, \ldots, k-1$. Clearly

$$
n \geq\left|A_{0} \cup \ldots A_{k-1}\right| \geq \sum_{i=0}^{k-1}\left|A_{i}\right|-\sum_{0 \leq i<j \leq k-1}\left|A_{i} \cap A_{j}\right|
$$

by the inclusion-exclusion formula. By our assumption $\sum_{i=0}^{k-1}\left|A_{i}\right| \geq k m$. The summand $\left|A_{i} \cap A_{j}\right|$ counts the number of disks contained in the intersection of the strips $i$ and $j$. This intersection is a rhombus whose area is

$$
F_{i j}=\frac{w^{2}}{\sin (j-i) \theta}
$$

Since the disks are pairwise disjoint,

$$
\left|A_{i} \cap A_{j}\right| \leq \frac{F_{i j}}{\pi}
$$

We thus have

$$
\sum_{0 \leq i<j \leq k-1}\left|A_{i} \cap A_{j}\right|=O\left(\sum_{0 \leq i<j \leq k-1} \frac{1}{\sin (j-i) \theta}\right) .
$$

The identity $\sin \alpha=\sin (\pi-\alpha)$ yields

$$
\sum_{0 \leq i<j \leq k-1} \frac{1}{\sin (j-i) \theta} \leq k \sum_{i=1}^{\lfloor k / 2\rfloor} \frac{1}{\sin i \theta}
$$

For $1 \leq i \leq k / 2$

$$
\frac{1}{\sin i \theta}=\frac{1}{\sin \frac{i \pi}{k}}=O\left(\frac{k}{i}\right) .
$$

Consequently the second sum in Equation (1) is bounded as follows:

$$
\sum_{0 \leq i<j \leq k-1}\left|A_{i} \cap A_{j}\right|=O\left(k^{2} \sum_{i=1}^{\lfloor k / 2\rfloor} \frac{1}{i}\right)=O\left(k^{2} \log k\right) .
$$


Let $c_{1}>0$ be an absolute constant such that $\sum_{0 \leq i<j \leq k-1}\left|A_{i} \cap A_{j}\right| \leq c_{1} \cdot k^{2} \log k$. Since $\log k \leq(\log n) / 2$ for $n \geq 16$, and using the above estimates, Equation (1) can be rewritten as

$$
n \geq m k-c_{1} \cdot k^{2} \log k \geq c_{2} \sqrt{n \log n} \sqrt{\frac{n}{\log n}}-2 c_{1} \frac{n}{\log n} \frac{\log n}{2}=\left(c_{2}-c_{1}\right) n .
$$

Take now $c_{2}=c_{1}+2$, and obtain $n \geq 2 n$ which is a contradiction.

Theorem 2. Given a pair of start and target configurations $S$ and $T$, consisting of $n$ congruent disks each, $\frac{3 n}{2}+O(\sqrt{n \log n})$ moves always suffice for transforming the start configuration into the target configuration. The entire motion can be computed in $O\left(n^{3 / 2}(\log n)^{-1 / 2}\right)$ time. On the other hand, there exist pairs of configurations that require $\left(1+\frac{1}{15}\right) n-O(\sqrt{n})$ moves for this task.

Proof. We start with the upper bound. Let $S^{\prime}$ and $T^{\prime}$ be the centers of the start disks and target disks, respectively, and let $\ell$ be the line guaranteed by Lemma 1 . Without loss of generality we can assume that $\ell$ is vertical. Denote by $s_{1}=\lfloor n / 2\rfloor$ and $s_{2}=\lceil n / 2\rceil$ the number of centers of start disks to the left and to the right of $\ell$. Let $m=O(\sqrt{n \log n})$ be the number of start disks contained in the vertical strip around $\ell$. Denote by $t_{1}$ and $t_{2}$ the number of centers of target disks to the left and to the right of $\ell$, respectively. By symmetry we can assume that $t_{1} \leq n / 2 \leq t_{2}$.

Let $R$ be a region containing all start and target disks (e.g., the smallest axis-aligned rectangle that contains all disks). The algorithm has three steps. All moves in the region $R$ are taken along horizontal lines, i.e., perpendicularly to the line $\ell$.

STEP 1 Slide to the far right all start disks whose centers are to the right of $\ell$ and the (other) start disks in the strip, one by one, in decreasing order of their $x$-coordinates (with ties broken arbitrarily). At this point all $t_{2} \geq n / 2$ target disks whose centers are right of $\ell$ are free.

STEP 2 Using all the $s_{1}^{\prime} \leq n / 2$ remaining disks whose centers are to the left of $\ell$, in increasing order of their $x$-coordinates, we fill free target positions to the right of $\ell$, in increasing order of their $x$-coordinates: each disk slides first to the left, then to the right on a wide arc and to the left again in the end. Note that $s_{1}^{\prime} \leq n / 2 \leq t_{2}$. Now all the target positions whose centers are to the left of $\ell$ are free.

STEP 3 Move to place the far away disks: first continue to fill target positions whose centers are to the right of $\ell$, in increasing order of their $x$-coordinates. When we are done, we fill target positions whose centers are to the left of $\ell$, in decreasing order of their $x$-coordinates. Note that at this point all target positions to the left of $\ell$ are "free."

The only non-target moves are those done in STEP 1 and their number is $n / 2+O(\sqrt{n \log n})$, so the total number of moves is $3 n / 2+O(\sqrt{n \log n})$. 
Algorithm. A trivial implementation of the algorithm examines all $k=\lceil\sqrt{n / \log n}\rceil$ strip directions each in $O(n)$ time, in order to find a suitable one, as described in the proof of Lemma 1. After that, $O(n \log n)$ time is spent for this direction for sorting and performing the moves. The resulting time complexity is $O\left(n^{3 / 2}(\log n)^{-1 / 2}\right)$.

Lower bound. The target configuration consists of a set of $n$ densely packed unit (radius) disks contained, for example, in a square of side length $\approx 2 \sqrt{n}$. The disks in the start configuration enclose the target positions in a ring-like structure with long "legs." Its design is more complicated and uses "rigidity" considerations as described below.

A packing $\mathcal{C}$ of unit (radius) disks in the plane is said to be stable if each disk is kept fixed by its neighbors [4]. More precisely, $\mathcal{C}$ is stable if none of its elements can be translated by any small distance in any direction without colliding with the others. It is easy to see that any stable system of (unit) disks in the plane has infinitely many elements. K. Böröczky [3] showed that there exist stable systems of unit disks with arbitrarily small density.

The main building block used in Böröczky's construction was a one-way infinite "bridge" made up of disks, which can be defined as follows. In Fig. 1, the initial section of such a one-way infinite bridge appears on the left of the vertical line $\ell$. Fix an $x-y$ rectilinear coordinate system in the plane. Let us start

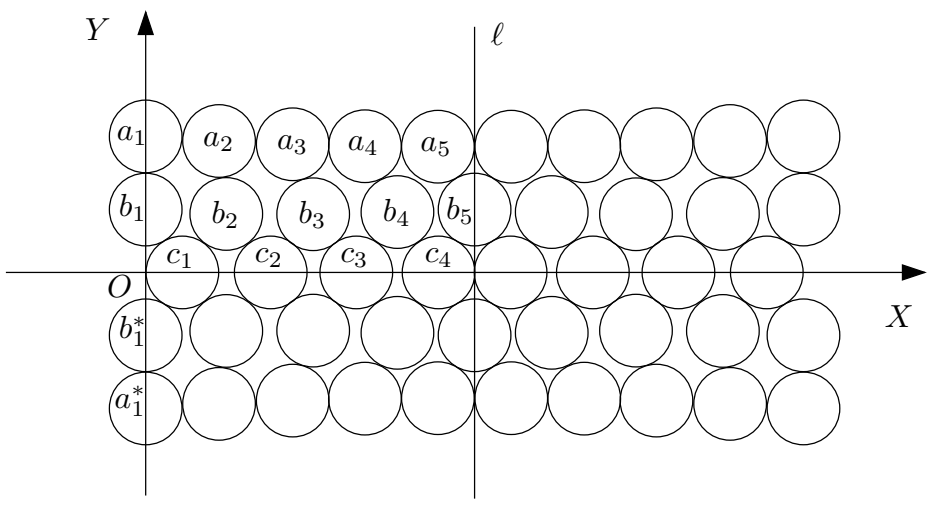

Fig. 1. A double bridge and its vertical line of symmetry $\ell$. The part left of $\ell$ forms the initial section of a one-way infinite bridge.

with five unit disks centered at

$$
a_{1}=(0,2+\sqrt{3}), b_{1}=(0, \sqrt{3}), c_{1}=(1,0), b_{1}^{*}=-b_{1}, \quad a_{1}^{*}=-a_{1},
$$

that serve as an "abutment." The bridge will be symmetric about the $x$-axis, so it is sufficient to describe the part of the packing in the upper half-plane. The set of centers of the disks is denoted by $C$. 
Take a strictly convex function $f(x)$ defined for all $x \geq 0$ such that $f(0)=$ $2+\sqrt{3}$ and $\lim _{x \rightarrow \infty} f(x)=2 \sqrt{3}$. Starting with $a_{1}$, choose a series of points $a_{2}, a_{3}, a_{4}, \ldots$ belonging to the graph of $f$ such that the distance between any two consecutive points satisfies

$$
\left|a_{i}-a_{i+1}\right|=2 \quad(i=1,2,3, \ldots) .
$$

All unit disks around these points belong to the packing, so that $a_{i} \in C$ for every $i$. These points will uniquely determine all other elements of $C$, according to the following rules.

Let $b_{2}$ be the point at distance 2 from both $c_{1}$ and $a_{2}$, which lies to the right of the line $c_{1} a_{2}$. Once $b_{2}$ is defined, let $c_{2}$ be the point on the $x$-axis, different from $c_{1}$, whose distance from $b_{2}$ is 2 . In general, if $b_{i}$ and $c_{i}$ have already been defined, let $b_{i+1}$ denote the point at distance 2 from both $c_{i}$ and $a_{i+1}$, lying on the right-hand side of their connecting line, and let $c_{i+1} \neq c_{i}$ be the (other) point of the $x$-axis at distance 2 from $b_{i+1}$. Let $C$, the set of centers of the disks forming the bridge, consist of all points $a_{i}, b_{i}, c_{i}(i=1,2,3, \ldots)$ and their reflections about the $x$-axis. Note that the points $c_{i} \in C$ lie on the $x$-axis, so they are identical with their reflections.

We need four properties of this construction, whose simple trigonometric proofs can be found in [3]:

1. the distance between any two points in $C$ is at least 2 ;

2. all unit disks around $a_{i}, b_{i}, c_{i} \quad(i=2,3,4, \ldots)$ are kept fixed by their neighbors;

3. all points $b_{2}, b_{3}, b_{4}, \ldots$ lie strictly below the line $y=\sqrt{3}$;

4. the $x$-coordinate of $c_{i}$ is smaller than that of $a_{i+1} \quad(i=1,2,3, \ldots)$.

It is not hard to see that the difference between the $x$-coordinates of $c_{i}$ and $a_{i+1}$ tends to zero as $i$ tends to infinity.

Next, we slightly modify the above construction. Take a small positive $\varepsilon$ and replace $f(x)$ by the strictly convex function

$$
f_{\varepsilon}(x):=(1+\varepsilon) f(x)-\varepsilon f(0)
$$

whose asymptote is the line $y=2 \sqrt{3}-(2-\sqrt{3}) \varepsilon$. Clearly, $f_{\varepsilon}(0)=f(0)$. If we carry out the same construction as above, nothing changes before we first find a point $a_{i}$ that lies below the line $y=2 \sqrt{3}$. However, if $\varepsilon$ is sufficiently large, sooner or later we get stuck: the construction cannot be continued forever without violating any of the conditions listed above. Let $k$ be the first integer for which such an event occurs, involving $a_{k}, a_{k+1}, b_{k}$, or $c_{k}$. By varying $\varepsilon>0$, it can be shown by a simple case analysis that the construction can be realized up to level $k$ so that the difference between the $x$-coordinates of $b_{k}$ and $a_{k}$ is 1 . It follows that the disk around $a_{k}$ is tangent to the vertical line $\ell$ passing through $b_{k}$. Remove the rightmost disk centered at $c_{k}$ from the set. Thus from the above condition, by taking the union of the part of $C$ built so far together with its reflection about $\ell$, we obtain the following: 
Lemma 2. There exist arbitrarily long finite packings ("double-bridges") consisting of five rows of unit disks, symmetric about the coordinate axes, in which all but eight disks are kept fixed by their neighbors. These eight exceptional disks are at the two abutments of the double-bridge and their $y$-coordinates are $\pm \sqrt{3}, \pm(2+\sqrt{3})$.

Notice that three such bridges can be connected at a "junction" depicted in Fig. 2 so that the angles between their "long" half-axes of symmetries (corresponding to the positive $x$-axis) are $\frac{2 \pi}{3}$. Consequently, using six double-bridges connected by six junctions one can enclose an arbitrarily large hexagonal region $H$. Let us attach a one-way infinite bridge to each of the unused sides of the junctions. As Böröczky pointed out, the resulting packing is stable.

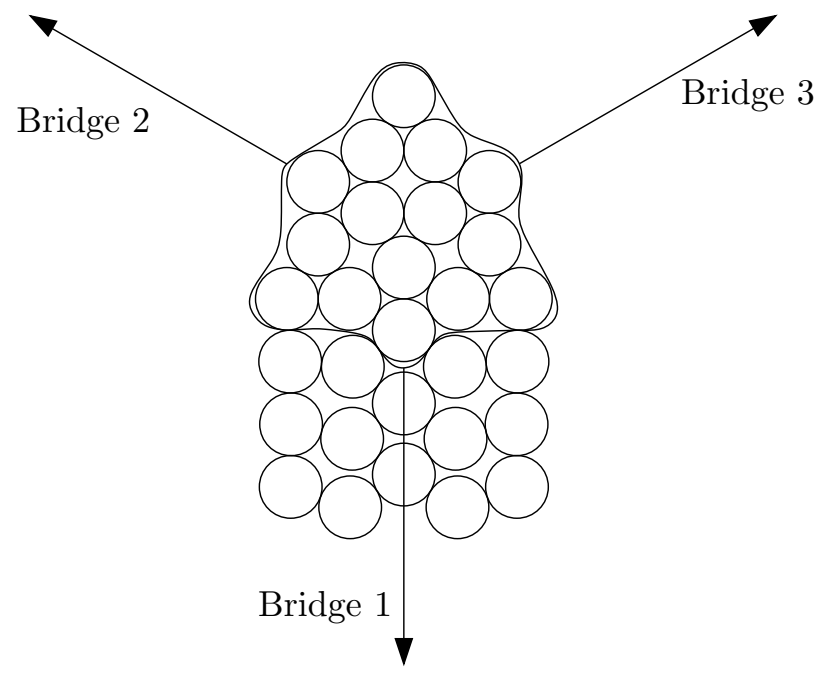

Fig. 2. Junction of type 1.

Let us refer to the disks in the start (resp. target) configuration as white (resp. black) disks. Now fix a large $n$, and take $n$ white disks. Use $O(\sqrt{n})$ of them to build six junctions connected by six double-bridges (as described above) to enclose a hexagonal region that can accommodate the $n$ nonoverlapping black disks. See also Fig. 3. Divide the remaining white disks into six roughly equal groups, each of size $\frac{n}{6}-O(\sqrt{n})$, and rearrange each group to form the initial section of a one-way infinite bridge attached to the unused sides ("ports") of the junctions. Notice that the number of necessary moves is at least $\left(1+\frac{1}{30}\right) n-$ $O(\sqrt{n})$. To see this, it is enough to observe, that in order to fill the first target, we have to break up the hexagonal ring around the black disks. That is, we have to move at least one element of the six double-bridges enclosing $H$. However, with the exception of the at most $6 \times 5=30$ white disks at the far ends of the truncated one-way infinite bridges, every white disk is fixed by its neighbours. 
Each of these bridges consists of five rows of disks of "length" roughly $\frac{n}{30}$, where the length of a bridge is the number of disks along its side. Therefore, before we could move any element of the ring around $H$, we must start at a far end and move a sequence of roughly $\frac{n}{30}$ white adjacent disks.

Instead of enclosing the $n$ black disks by a hexagon, we can construct a triangular ring $T$ around them, consisting of three double-bridges (see Fig. 3). To

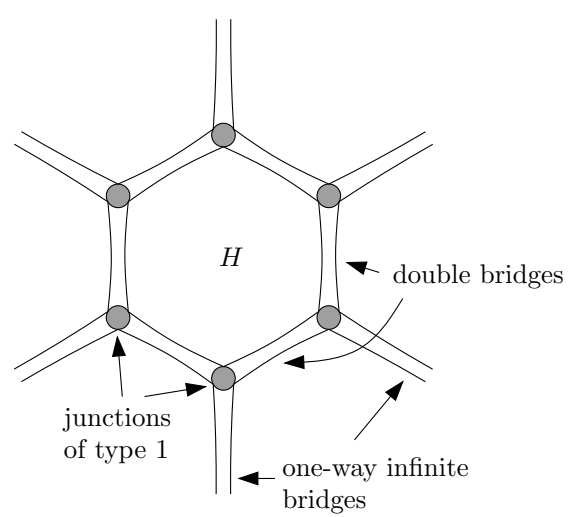

(a)

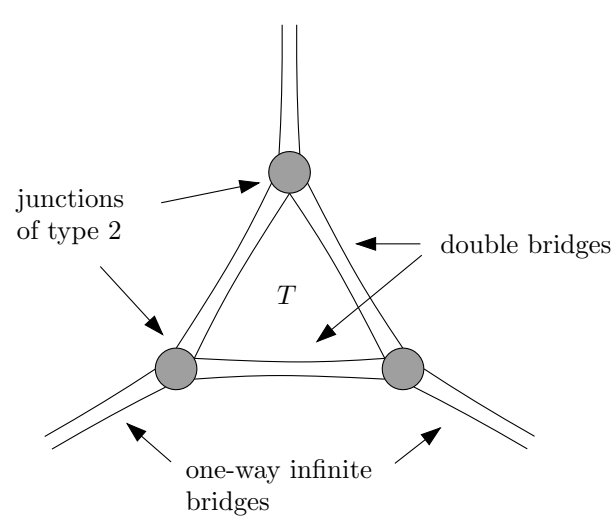

(b)

Fig. 3. Two start configurations based on hexagonal and triangular rings.

achieve this, we have to build a junction of three sides establishing a connection between the abutments of three bridges such that the angles between their halfaxes of symmetry are $\frac{5 \pi}{6}, \frac{5 \pi}{6}$, and $\frac{\pi}{3}$. Such a junction is shown on Fig. 4. The convex hull of the disk centers (for the disks in the junction) is a pentagon symmetric with respect to a vertical line passing through the top vertex. Four out of the five centers along each of the three sides of the pentagon connected to bridges are collinear. The disk centers on the other two sides form two slightly concave chains. The number of necessary moves is at least $\left(1+\frac{1}{15}\right) n-O(\sqrt{n})$ for this second construction. This completes the proof of Theorem 2.

Remarks. We believe that our lower bound in Theorem 2 is closer to the truth. Closing the gap between the bounds remains an interesting problem which seems to require new ideas.

Note that moving out in STEP 1 only start disks whose centers are right of $\ell$ and those disks intersecting $\ell$ would not necessarily free all targets whose centers are right of $\ell$. This is the reason for working with a strip of width 6 around $\ell$; in fact imposing a bound on the number of disks contained in a strip of width 4, which extends three units to the left of $\ell$ and one unit to the right of $\ell$ would be enough. 


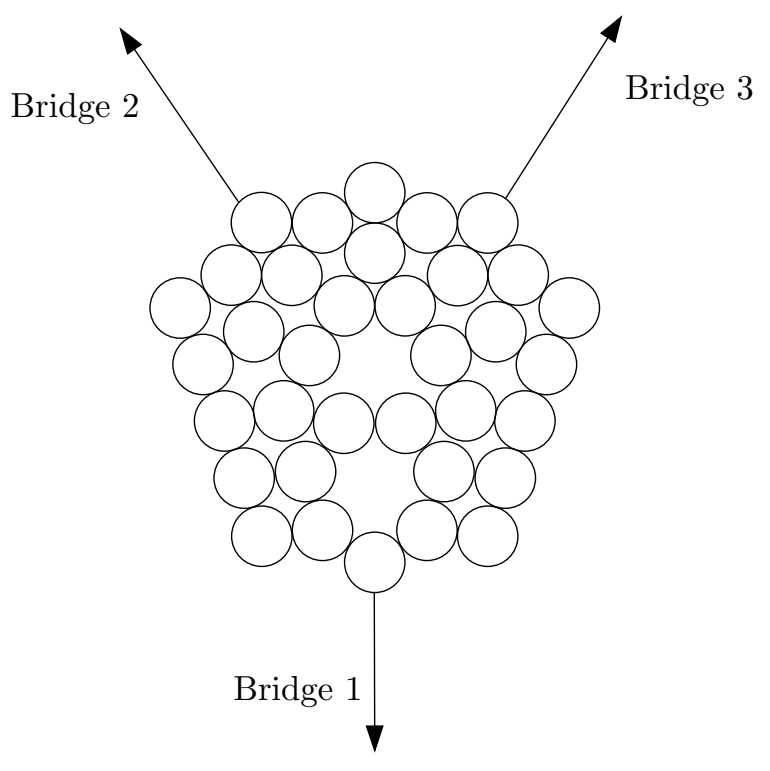

Fig. 4. Junction of type 2 .

\section{Arbitrary disks}

For the reconfiguration of systems of arbitrary disks we obtain tight bounds (modulo lower order terms):

Theorem 3. Given a pair of start and target configurations, consisting of $n$ disks of arbitrary radii each, $2 n$ moves always suffice for transforming the start configuration into the target configuration. The entire motion can be computed in $O(n \log n)$ time. On the other hand, there exist pairs of configurations that require $2 n-o(n)$ moves for this task, for every sufficiently large $n$.

Proof. The upper bound is immediate, using the universal reconfiguration algorithm described above. The recursive lower bound construction is depicted in Figure 6 . The basic construction in Figure 5 (which will be repeated recursively) gives a lower bound of $\approx 3 n / 2$ : it consists of a large disk surrounded by $n-1$ small disks, whose centers form a regular polygon with $n-1$ vertices (let $n$ be even). The target configuration has all small disks inside the original big disk and the large disk somewhere else. No small disk target can be filled before the large disk moves away, that is, before roughly half of the $n-1$ small disks move away. So about $3 n / 2$ moves in total are necessary.

The recursive construction is obtained by replacing the small disks around a big one by the "same" construction scaled (see Figure 6). To make it work we choose: 1) all disks of distinct radii, and 2) the small disks on the last level or recursion have targets inside the big ones they surround (the other disks have targets somewhere else). Since all disks have distinct radii, one can think of them 

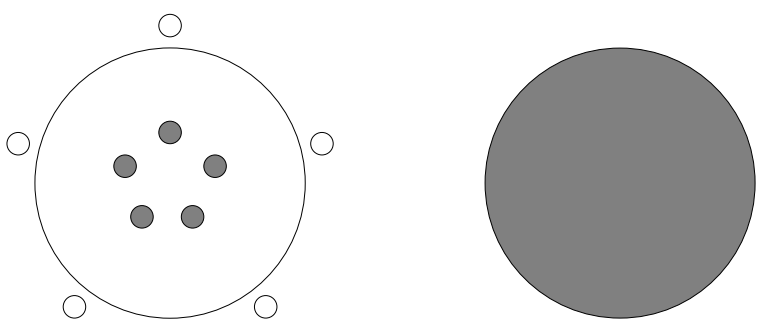

Fig. 5. A simple lower bound construction (basic step for the recursive construction) for sliding disks of arbitrary radii.

as being labeled. If there are $k$ levels in the recursion, about $n / 2+n / 4+\cdots+n / 2^{k}$ non-target moves are necessary. The precise calculation follows.
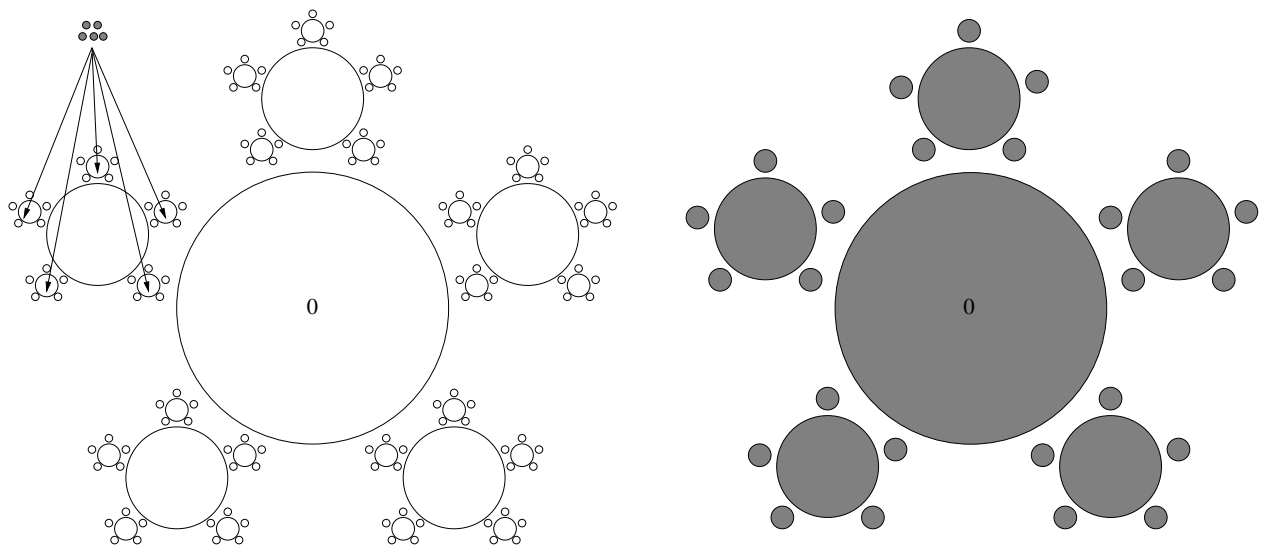

Fig. 6. Recursive lower bound construction for sliding disks of arbitrary radii: $m=2$ and $k=3$.

There is one large disk labeled 0 , and $2 m+1$ groups of smaller disks around it close to the vertices of a regular $(2 m+1)$-gon $(m \geq 1)$. Let $m$ be fixed, and $k$ be the number of levels in the recursion ( $m$ and $k$ will be chosen later). Let $n=N(k)$ be the number of disks in the set, and $x=X(k)$ be the number of non-target moves performed (at level $k$ ). We have

$$
N(0)=1, \quad X(0) \geq 0, \quad N(1)=2 m+2, \quad X(1) \geq m .
$$

$N(k)$ and $X(k)$ satisfy the following recurrences:

$$
N(k)=(2 m+1) N(k-1)+1,
$$

$X(k) \geq m N(k-1)+(m+1) m N(k-2)+\ldots+(m+1)^{k-2} m N(1)+(m+1)^{k-1} m$. 
The first recurrence gives

$$
N(k)=(2 m+1)^{k}+\ldots+(2 m+1)+1=\frac{(2 m+1)^{k+1}-1}{2 m} .
$$

Plugging this into the inequality for $X(k)$ yields

$$
X(k) \geq m \sum_{i=0}^{k-1} \frac{(2 m+1)^{k-i}-1}{2 m}(m+1)^{i}=\frac{1}{2} \sum_{i=0}^{k-1}\left((2 m+1)^{k-i}-1\right)(m+1)^{i} .
$$

Using standard manipulations, the above inequality becomes

$$
X(k) \geq \frac{(2 m+1)^{k+1}-2(m+1)^{k+1}+1}{2 m} .
$$

This can be rewritten as

$$
X(k) \geq \frac{(2 m+1)^{k+1}-1-2(m+1)^{k+1}+2}{2 m}=n-\frac{(m+1)^{k+1}-1}{m} .
$$

Put

$$
z=\frac{(m+1)^{k+1}-1}{m}
$$

Then

$$
\frac{z}{n}=2 \frac{(m+1)^{k+1}-1}{(2 m+1)^{k+1}-1} \leq 2\left(\frac{m+1}{2 m+1}\right)^{k+1} \rightarrow 0, \text { for } k \rightarrow \infty
$$

Thus $n+x \geq 2 n-z=2 n-o(n)$ and the lower bound follows for $n=N(k)$. The same result carries over for all sufficiently large $n$. In particular for $m=1$, we get $n+x=2 n-O\left(n^{\log _{3} 2}\right)=2 n-O\left(n^{0.631}\right)$.

Acknowledgement. The authors thank Jan Siwanowicz for his valuable remarks and for many interesting conversations on the topic.

\section{References}

1. M. Abellanas, F. Hurtado, A. G. Olaverri, D. Rappaport, and J. Tejel, Moving coins. Short version in Abstracts of Japan Conference on Discrete and Computational Geometry, 2004. Full version submitted to LNCS Proceedings.

2. N. Alon, M. Katchalski, and W. R. Pulleyblank, Cutting disjoint disks by straight lines, Discrete $\mathcal{G}$ Computational Geometry, 4 (1989), 239-243.

3. K. Böröczky, Über stabile Kreis- und Kugelsysteme (in German), Ann. Univ. Sci. Budapest. Eötvös Sect. Math. 7 (1964), 79-82.

4. P. Brass, W. O. J. Moser, and J. Pach, Research Problems in Discrete Geometry, Springer-Verlag, 2005, to appear.

5. N. G. de Bruijn, Aufgaben 17 and 18 (in Dutch), Nieuw Archief voor Wiskunde 2 (1954), 67. 
6. E. Demaine, M. Demaine, and H. Verrill, Coin-moving puzzles, in More Games of No Chance, edited by R. J. Nowakowski, pp. 405-431, Cambridge University Press, 2002.

7. A. Dumitrescu and J. Pach, Pushing squares around, Proceedings of the 20-th Annual Symposium on Computational Geometry, (SOCG'04), NY, June 2004, 166123.

8. L. Fejes Tóth and A. Heppes, Über stabile Körpersysteme (in German), Compositio Mathematica, 15 (1963), 119-126.

9. L. Guibas and F. F. Yao, On translating a set of rectangles, in Computational Geometry, F. Preparata (ed.), pp. 61-67, Vol. 1 of Advances in Computing Research, JAI Press, London, 1983. 